\title{
Growth of Yeast Colonies on Solid Media
}

\author{
By RAVINDRANATH S. KAMATH AND HENRY R. BUNGAY* \\ Department of Chemical Engineering, Rensselaer Polytechnic Institute, Troy, NY 12181, USA
}

(Received 3 March 1988; revised 1 July 1988)

\begin{abstract}
Colonies on nutrient agar of the aerobic yeast Candida utilis show linear increases in diameter and height with time throughout most of the growth cycle. The concentration of glucose in the agar has a negligible effect on radial growth rate although an increase in the glucose concentration prolongs the linear radial growth phase. The rate of increase in height of the colony is proportional to the square root of the initial glucose concentration. A new model that considers both glucose diffusion and oxygen diffusion in the colony is consistent with the observed colony profiles.
\end{abstract}

\section{INTRODUCTION}

There have been numerous studies of colonies of bacteria and filamentous fungi but little attention has been given to yeast colonies. Pirt (1967) formulated the physical and chemical factors affecting the growth rate of colonies (mainly bacterial) on solid media; since then there have been several experimental studies (Cooper et al., 1968; Johnson et al., 1970; Palumbo et al., 1971; Rieck et al., 1973; Burchard, 1974; Pirt, 1975; Reyolle \& Letellier, 1979; Wimpenny, 1979; Wimpenny \& Parr, 1979; Lewis \& Wimpenny, 1981). Wimpenny \& Lewis (1977) used actual oxygen consumption rates to predict the penetration depth of oxygen into the colony. Oxygen profiles in colonies and in the solid medium have been measured using microelectrodes (Wimpenny \& Coombs, 1983; Bungay et al., 1983; Peters et al., 1987). Fraleigh \& Bungay (1986) modelled oxygen and substrate gradients in the colony.

Wimpenny (1979) found that near the periphery or leading edge of a growing colony, the height rises relatively steeply to a ridge. Beyond the point of inflexion marking this ridge, the height rises much less steeply to a flat or domed centre. He proposed that growth is exponential and unrestricted by diffusion in young colonies and in the leading edge region of older colonies. At the change in the slope of the profile near the ridge between the two areas, nutrient becomes less available possibly because of depletion of a narrow layer adjacent to the colony base. The growth rate in this area depends on the rate of diffusion of one or more growth limiting nutrients through the agar. Lewis \& Wimpenny (1981) also reported variation in colony size and growth rates due to oxygen availability and different nutritional conditions.

According to Pirt (1967), when nutrients have a long diffusion path to the top of a colony, growth is restricted to the outer peripheral zone. However, Palumbo et al. (1971) found an increase in colony height throughout the entire growth cycle. There are several similar observations (Wimpenny, 1979; Wimpenny \& Parr, 1979; Lewis \& Wimpenny, 1981). Reyrolle \& Letellier (1979) found two zones of growth - a peripheral zone responsible for the increase in diameter and a central zone that accounts for the increase in height. In the peripheral zone, the main factor for growth was bacterial mobility.

An approximately linear increase in colony height, which later slows down, has been reported to occur during the earlier period of growth (Palumbo et al., 1971; Wimpenny, 1979; Lewis \& Wimpenny, 1981). Pirt (1967) postulated that at steady state, the width of the growing zone would remain constant and hence the radial growth rate would become constant. This is called the 'linear" or 'radial' growth law and has been observed by many other workers. This phase is 
found to continue until the colony reaches about $80 \%$ of its final size. The linear growth phase eventually switches to a slower phase in which the colony area increases linearly with time (Cooper et al., 1968). It was postulated that the onset of strict diffusional resistances caused by nutrient depletion is responsible for this phase and may explain the morphological changes observed in older colonies.

At very low glucose concentrations $(0 \cdot 01-0 \cdot 1 \%)$, Rieck et al. (1973) found that colonies exhibited transition points or times at which the initial linear phase changed to a second and slower linear phase. As the concentration of glucose was lowered, colonies had earlier transition points and slower radial growth rates. Growth of the colonies after the transition point was shown to be limited by the rate of diffusion of glucose and by its availability to the colony.

Gray \& Kirwan (1974) studied the effect of the boundary walls on the growth of colonies of Saccharomyces cerevisiae by varying the size of the culture dish. Transition from radial to area growth rate laws did indeed relate to nutrient depletion. In addition, growth rate was independent of the size and shape of the inoculum and it was concluded that the radial growth law holds for longer periods of time in larger dishes.

Jones \& Gray $(1978 a, b, 1979)$ exposed yeast colonies on a gel to a continuously flowing nutrient solution from below, resulting in a constant nutrient concentration at the colony-gel interface. Rather than confirming the property of indefinite linear-radial growth, maturing colonies in this apparatus showed an exponential increase in diameter with time. An eventual departure from exponential growth was observed and attributed to such factors as buildup of toxic materials (ethanol) and depletion of oxygen and glucose.

For both bacterial and yeast colonies there is a minimun substrate concentration below which no growth occurs, and a linear relationship between the growth rate, $k_{\mathrm{r}}$, and the square-root of the substrate concentration (Pirt, 1967). The threshold concentration seems to be peculiar to bacterial colony growth and is not found with filamentous fungi (Pirt, 1975). Colonies of filamentous fungi or moulds are inherently different from those of bacteria and yeast because of hyphal extensions. Bull \& Trinci (1977) and Prosser (1982) offer excellent reviews.

Pirt (1967) derived equations for the linear growth law and the area law

$$
\begin{gathered}
r=\mu w t+r_{0} \\
r^{2}=2 \mu b t+r_{0}^{2}
\end{gathered}
$$

where $r=$ the radius of the colony, $r_{0}=$ the initial radius, $\mu=$ the specific growth rate coefficient, $w=$ the width of peripheral growth zone, $b=\mathrm{a}$ constant and $t=$ time. The main assumptions are constant colony height, growth only in the periphery, and that the cross-section of the growing zone is rectangular.

The above model is not strictly valid, but the rate of increase in colony height is much smaller than the radial growth rate so that the model fits actual data well. Palumbo et al. (1971) modelled the colony as a spherical segment. This choice is reasonably accurate for small colonies. The radius $R$ of the sphere of which a colony of radius $r$ and height $h$ is a segment can be calculated using the formula

$$
R=\frac{\left(r^{2}+h^{2}\right)}{2 h}
$$

and the colony volume is given by

$$
V=\frac{1}{6} \pi h\left(3 r^{2}+h^{2}\right)
$$

Using the same assumption, Wimpenny \& Coombs (1983) calculated the volume of the aerobic surface layer, i.e. the growing zone of a colony $\left(V_{0}\right)$ as

$$
V_{0}=\frac{\pi}{6}\left\{3(h+r)^{2} d-6(h+r) d^{2}+4 d^{3}\right\}
$$

where $d$ is the depth of oxygen penetration into the colony. 
The gradual changes in a colony with time permit the assumption that at any time a pseudosteady-state exists with regard to the gradients of nutrients. Mass continuity equations governing the gradients of nutrients within the microbial colony can be described by

$$
\nabla \cdot\left(D_{\mathrm{i}} \nabla c_{\mathrm{i}}\right)=Q_{\mathrm{i}}
$$

where $c, Q$ and $D$ are the concentration, rate of consumption and mass diffusivity of species $\mathrm{i}$ respectively, provided the appropriate boundary conditions are specified.

Gray \& Kirwan (1974) modelled colony growth with a sink term for glucose (the limiting nutrient) of the form

$$
Q=\mu n\left(s-s_{\mathrm{i}}\right)
$$

where $n$ is the concentration of the organisms, $s$ is the concentration of the substrate and $s_{\mathrm{i}}$ is the threshold concentration. They considered the one-dimensional propagation of a colony, in the $x$ direction, the plane front of the colony moving with a velocity $K_{\mathrm{r}}$ to develop the equation

$$
K_{\mathrm{r}}=k \sqrt{c D} \sqrt{s_{0}-s_{\mathrm{i}}} \sqrt{\mu}
$$

where $k$ is a constant. This model has the following drawbacks: (a) its one-dimensional nature; (b) the assumption of only one growth-limiting nutrient; and (c) the assumed relation for the consumption rate of the substrate is overly simplistic to be truly applicable to a biological system.

Colonies of aerobic species receive glucose (carbon and energy source) from the medium below while oxygen (terminal electron acceptor) diffuses from the gaseous phase. The counterdiffusion and uptake of these two species means that there is a possibility that in certain regions, metabolism is limited by oxygen availability while in others, glucose is lacking. Fraleigh (1985) sought to overcome the limitations of the model of Gray and Kirwan by simulating oxygen and glucose gradients within a microbial colony using a more realistic two-dimensional approach and also considering double-substrate (glucose and oxygen) limited growth kinetics. He too used the spherical segment approximation of the colony shape.

In this paper we describe the growth on nutrient agar of colonies of the aerobic yeast Candida utilis.

\section{METHODS}

Candida utilis (ATCC 42416) was grown at $25^{\circ} \mathrm{C}$ in $500 \mathrm{ml}$ shake flasks with $150 \mathrm{ml}$ of medium comprising Dglucose $\left(10 \mathrm{~g} \mathrm{l}^{-1}\right)$, peptone $\left(5 \mathrm{~g}^{-1}\right)$ and yeast extract $\left(5 \mathrm{~g} \mathrm{l}^{-1}\right)$, sterilized together for $20 \mathrm{~min}$ at $121^{\circ} \mathrm{C}$. For growth on Petri dishes (10 cm diameter, $1.5 \mathrm{~cm}$ high), medium containing the same concentrations of peptone and yeast extract was solidified with $15 \mathrm{~g}_{\text {agar }} \mathrm{l}^{-1}$; the glucose concentration used was $1,5,8,10,15$ or $20 \mathrm{~g}^{-1}$. This range was chosen because, in another study of yeast colonies (Gray \& Kirwan, 1974), the threshold concentration was found to be $2.4 \mathrm{~g} \mathrm{l}^{-1}$, and beyond about $12 \mathrm{~g} \mathrm{l}^{-1}$ there was a departure from the linear relationship between $K_{\mathrm{r}}$ and $\sqrt{s_{0}}$. The Petri dishes were inoculated to give $20-25$ colonies per plate. Growth was at $25^{\circ} \mathrm{C}$.

The colonies were measured with a probe (electrode) and micromanipulator. A model $610 \mathrm{C}$ solid state electrometer (Keithley Instruments) and a voltage source were connected to a reference electrode of silver coated with $\mathrm{AgCl}$ and an ordinary sewing needle that served as the probe. The Petri dish was held in position on the micromanipulator stand by a small amount of putty at the edges. The reference electrode was embedded into the solid agar medium, and the probe (needle) was centred over a selected colony. A reference point was established above the agar surface, and the probe was lowered to contact the agar along the line of measurement near the ends of the colony to determine the inclination of the agar. Touching either the colony of the agar completed the electrical circuit and caused a current to pass through the electrometer. Colony diameter was measured in two perpendicular directions and averaged.

\section{RESULTS AND DISCUSSION}

Typical colony profiles at various times after inoculation on different glucose concentrations are shown graphically in Fig. 1. None of the colonies was completely flat even after long growth periods and most had reasonably circular profiles. A common feature was a point of inflexion close to the edge of the colony, as observed by Wimpenny (1979). The profiles at different times were reasonably parallel, at least during the period when the colony height was increasing. 


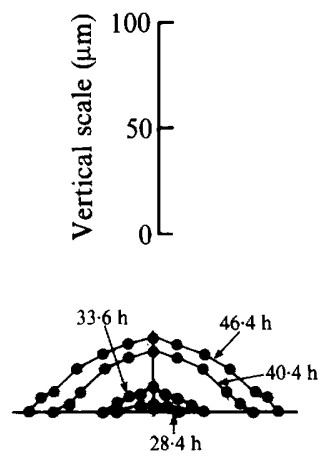

$1 \mathrm{~g}$ glucose $1^{-1}$

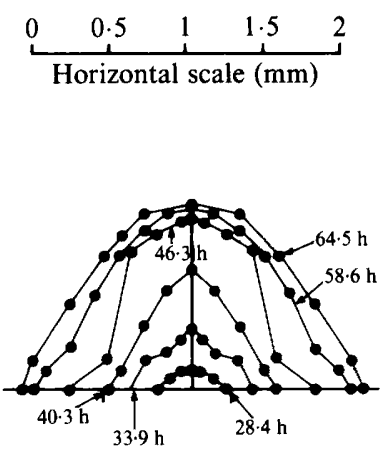

$8 \mathrm{~g}$ glucose $1^{-1}$

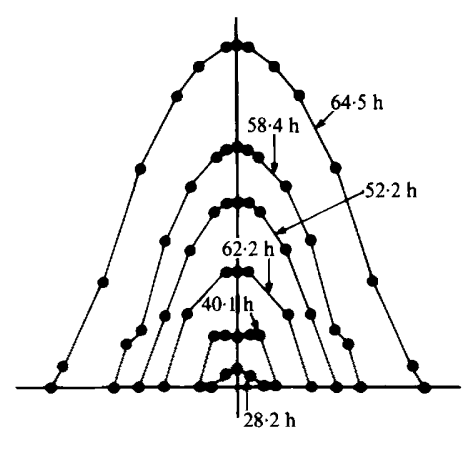

$20 \mathrm{~g}_{\text {glucose }} \mathrm{1}^{-1}$

Fig. 1. Typical measured colony profiles at various times on nutrient agar with glucose.

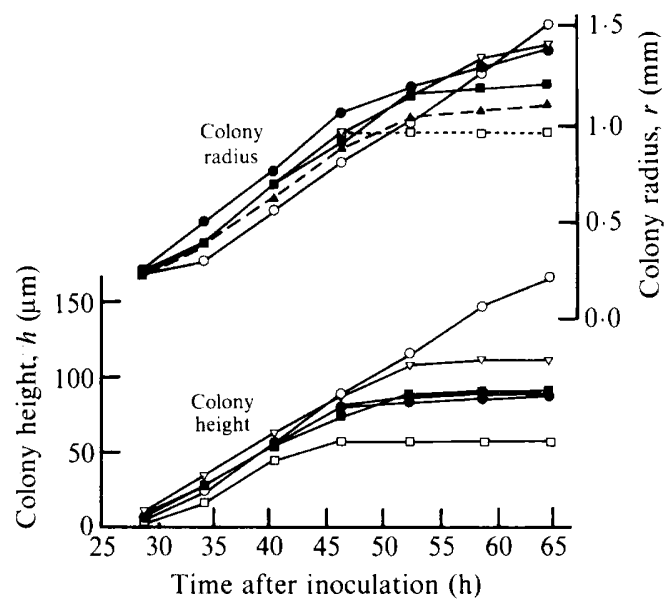

Fig. 2. Measured colony dimensions versus time at selected glucose concentrations $\left(\square, 1 \mathrm{~g}^{-1} ; \boldsymbol{\Delta}, 5 \mathrm{~g}\right.$ $\left.\mathrm{I}^{-1} ; 8 \mathrm{~g} \mathrm{l}^{-1} ; \mathbf{0}, 10 \mathrm{~g} \mathrm{l}^{-1} ; \nabla, 15 \mathrm{~g} \mathrm{l}^{-1} ; 0,20 \mathrm{~g} \mathrm{l}^{-1}\right)$.

Colony radius increased linearly with time at all glucose concentrations (upper portion of Fig. 2), but the findings of Pirt (1967) and Gray \& Kirwan (1974) of a radial growth rate proportional to the square of the rate of substrate concentration were not confirmed. We postulate that glucose concentration at the periphery of our colonies was above the value of the half-saturation constant until a colony was large enough to deplete a sizeable region of the agar.

There was no systematic trend in the variation of radial growth rate, $K_{\mathrm{r}}$, with $s_{0}$. In fact, the $K_{\mathrm{r}}$ values were much the same. The linear phase extended over a longer time period as the glucose concentration was increased. This is to be expected since the eventual departure from linearity is caused by glucose depletion. For the case of $8 \mathrm{~g}$ glucose $\mathrm{l}^{-1}$, two linear phases were observed as was reported earlier by Rieck et al. (1973), but only at very low glucose concentrations. The constancy of $K_{\mathrm{r}}$ with respect to $s_{0}$ is not entirely surprising. In the linear phase, growth is assumed to be restricted to the peripheral region (Pirt, 1967), where the concentration of glucose is nearly equal to $s_{0}$. Hence, unless $s_{0}$ itself is very small, glucose will not be a limiting factor.

Colony height increased linearly with time over most of the growth period at all glucose concentrations studied (lower portion of Fig. 2). Although the data were erratic, there is a definite increasing trend of rate of height increase, $K_{\mathrm{h}}$, with glucose concentration. 
Development of a mathematical model

The shape of the colony is assumed to be a spherical segment (Palumbo et al., 1971; Wimpenny \& Coombs, 1983). This implies that the profiles at various times will be parallel as was observed in this study. The radius of the sphere bounding the segment is given by equation 3. The following relationship holds

$$
\mathrm{d} h=\mathrm{d} r \cos \theta
$$

where

$$
\cos \theta=\frac{r}{R}=\frac{2 r h}{r^{2}+h^{2}}
$$

and

$$
\frac{\mathrm{d} r}{\mathrm{~d} h}=\frac{r^{2}+h^{2}}{2 r h}
$$

Substituting $r=v h$ leads to

$$
\frac{\mathrm{d} h}{h}=\frac{2 v \mathrm{~d} v}{1-v^{2}}
$$

On integrating the above expression and simplifying, the following solution is obtained

$$
h\left(v^{2}-1\right)=\text { constant }=k
$$

Hence, the final equation for the colony profile is:

$$
\frac{r^{2}-h^{2}}{h}=k=\frac{r_{0}^{2}-h_{0}^{2}}{h_{0}}
$$

which is the equation of a hyperbola. This can be rewritten in the following forms

$$
\begin{gathered}
r=\sqrt{h(h+k)} \\
h=\frac{k}{2}\left\{\sqrt{1+(2 r / k)^{2}}-1\right\}
\end{gathered}
$$

Thus, by specifying the value of the parameter $k$, the colony profile is determined. The value of $k$ depends on the species of micro-organism and many other factors (Jones et al., 1978a, b, 1979).

Case of constant glucose concentration at the agar surface. The assumptions are as follows. (a) The thickness to which a diffusing nutrient can penetrate into the colony is given by the following equation derived by Pirt (1967)

$$
h=\sqrt{2 D s_{0} / q}
$$

where $h$ is the colony height, $D$ is the diffusion coefficient, $s_{0}$ is the concentration of nutrient at the surface, and $q$ is the metabolic coefficient ( $\mathrm{g}$ nutrient consumed per $\mathrm{cm}^{3}$ of colony per second). (b) Since $s_{0}$ is constant for both the nutrients glucose and oxygen, the depth, $d$, of penetration of oxygen into the colony as well as the height, $h_{9}$, to which glucose from the solid medium can diffuse are assumed to be constant. (c) It is further known that $d$ is less than $h_{\mathrm{g}}$ (Pirt, 1967).

Fig. 3 portrays growing zones in a colony as shaded areas. Four phases can be distinguished in the growth of the colony, depending on the height of the colony.

Phase $1(h \leqslant d)$. When the colony height is less than the oxygen penetration depth, $d$, the entire colony grows exponentially (Fig. 3A), since both glucose and oxygen are available throughout the colony. This is the initial exponential phase predicted by Pirt (1967).

Phase $2\left(d<h \leqslant h_{\mathrm{g}}\right)$. When the colony height increases beyond $d$ and until it reaches $h_{9}$, glucose is available throughout the colony, but oxygen is available only on the colony surface resulting in an annular region of constant thickness $d$, which is the growth zone in this phase (Fig. 3B). 
A
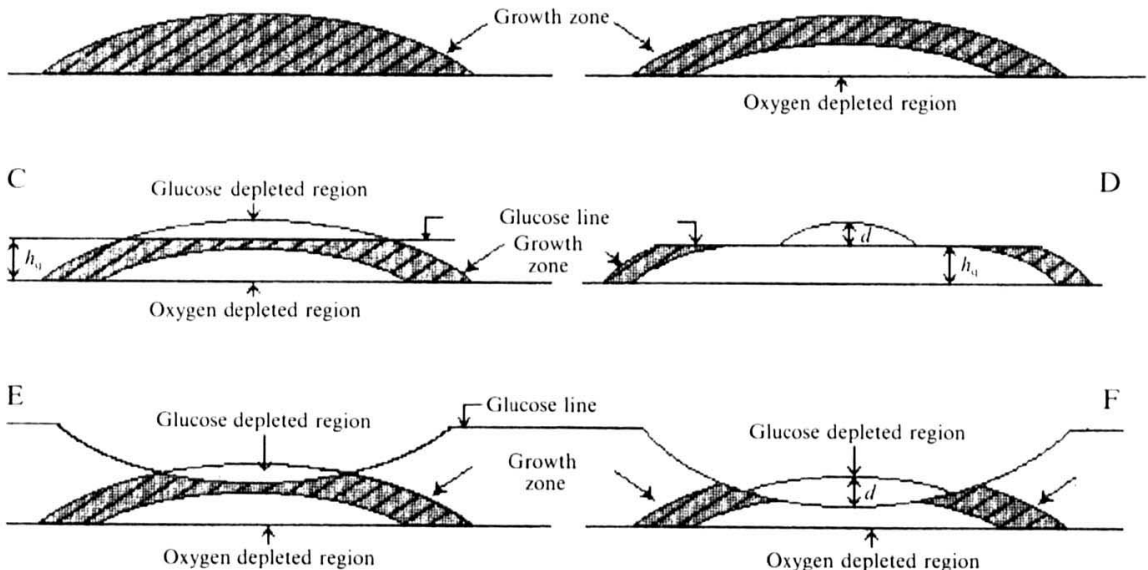

Fig. 3. Growth phases in a colony. A, Growth throughout colony, no limitation. B, No growth in regions remote from air. C, Growth in top portion limited by glucose, lower portion limited by oxygen. D, Growth only at periphery; overlap of limitations. E, More severe glucose depletion when not supplied continuously. F, Glucose not supplied continuously; overlap of limitations.

Phase $3\left(h_{\mathrm{g}}<h<h_{\mathrm{g}}+d\right)$. When the colony height increases beyond $h_{\mathrm{g}}$, there are regions in the colony which are deficient in glucose (at the top of the colony) as well as regions where oxygen cannot diffuse. This leads to a growth zone in the form of a truncated annular spherical segment (Fig. 3C). This phase continues until the colony height reaches a value of $\left(h_{\mathrm{g}}+d\right)$, at which point the lower growth front touches the glucose line (i.e. the hypothetical line which defines the maximum height to which glucose can diffuse). At this height, there is no point on the colony centre line where both glucose and oxygen are adequate. Of course, reduced glucose or oxygen does not necessarily halt growth completely, and slow growth in the limited zones will distort the colony from the shape predicted by this simplified model. Hence, it is proposed that the final height of the microbial colony is equal to $\left(h_{g}+d\right)$.

Phase $4\left(h=h_{\mathrm{g}}+d\right)$. In this phase, the colony height remains constant at the value $\left(h_{\mathrm{g}}+d\right)$. Here, only the outer peripheral region of constant thickness $d$ and constant height $h_{\mathrm{g}}$ is the growth zone (Fig. 3D). Thus, we can see that this model predicts the experimentally observed point of inflexion at height $h_{9}$, since the portion above the glucose line remains as a spherical segment while the lower portion expands outwards.

The advantage of the spherical segment assumption is that the mathematical formulation becomes relatively simplified although numerical solutions are still required. If the density of biomass is assumed to be constant, the equation for colony growth (equation 17) can be rewritten as

$$
\frac{\mathrm{d} V}{\mathrm{~d} t}=\mu V_{\mathrm{g}}
$$

where $V$ is the total colony volume and $V_{g}$ is the volume of the growth zone.

Up to the end of phase 3, the colony shape is that of a spherical segment. Hence, for phases $1-$ 3 , the total colony volume $V$ is given by equation 9 . In phase $1, V_{\mathrm{g} 1}$ is equal to $V$. In phase 2 , the volume of the growth zone $V_{\mathrm{g} 2}$ is given by

$$
V_{\mathrm{g} 2}=\frac{\pi}{6}\left[h\left(3 r^{2}+h^{2}\right)-(h-d)\left\{(h-d)^{2}+3(h-d)(h-d+k)\right\}\right]
$$

The volume of the growth zone in phase 3 can be derived as

$$
V_{93}=V_{92}-\frac{\pi}{6}\left(h-h_{\mathrm{g}}\right)\left\{\left(h-h_{9}\right)^{2}+3\left(h-h_{\mathrm{g}}\right)\left(h+h_{\mathrm{g}}+k\right)\right\}
$$


The volume of the growth zone in phase 4 is the volume of the segment of constant height $h_{\mathrm{g}}$ and constant thickness $d$. Finally, the total colony volume in phase 4 is equal to the constant volume of the spherical segment of height $\left(h_{g}+d\right)$, which is the volume at the end of phase 3 , plus the volume of the truncated annular segment of constant height $h_{9}$ and thickness $\left(r-r_{3}\right)$, where $r_{3}$ is the radius at the end of phase 3 obtained by using $h=\left(h_{\mathrm{g}}+d\right)$ in the relation between $h$ and $r$ (equation 16). Thus, substituting these expressions for $V$ and $V_{g}$ for the various phases into the growth equation (equation 20), the following equations are obtained, which can be solved in conjunction with the relationship between $r$ and $h$ (equations 14, 18 and 19).

Phase 1.

$$
3\left(r^{2}+h^{2}\right) \frac{\mathrm{d} h}{\mathrm{~d} t}=\mu\left(3 r^{2}+h^{2}\right)
$$

An analytical solution leads to the following relation between $h$ and $t$

$$
\ln \left[h^{2}(4 h+3 k) /\left\{h_{0}^{2}\left(4 h_{0}+3 k\right)\right\}\right]=\mu t
$$

The colony radius $r$ is related to $t$ using equation 18 .

Phase 2.

$$
12 r h \frac{\mathrm{d} r}{\mathrm{~d} t}=\mu V_{\mathrm{g} 2}
$$

where $V_{92}$ is given by equation 21 .

Phase 3.

$$
12 r h \frac{\mathrm{d} r}{\mathrm{~d} t}=\mu V_{93}
$$

where $V_{\mathrm{g} 3}$ is given by equation 22 .

Phase 4. Substituting for $V$ and $V_{9}$ into equation 18 and simplifying produces the following equation

$$
\begin{gathered}
6\left[r f+\frac{\mathrm{d} f}{\mathrm{~d} r}\left\{r^{2} / 2+5 f^{2} / 2+\left(k-2 h_{\mathrm{g}}\right) f-k h_{\mathrm{g}}\right\}\right] \frac{\mathrm{d} r}{\mathrm{~d} t}=\mu\left[f \left[3 r^{2}+f^{2}+\left(f-h_{\mathrm{g}}\right)\right.\right. \\
\left\{\left(f-h_{\mathrm{g}}\right)^{2}+3\left(f-h_{\mathrm{g}}\right)\left(f+h_{\mathrm{g}}+k\right)\right\}-\left\{(f-d)\left((f-d)^{2}+3(f-d)(f-d+k)\right)\right\} \\
\left.\left.\quad+\left(f-d-h_{\mathrm{g}}\right)\left\{\left(f-d-h_{\mathrm{g}}\right)^{2}+3\left(f-d-h_{\mathrm{g}}\right)\left(f-d+h_{\mathrm{g}}+k\right)\right\}\right]\right]
\end{gathered}
$$

The equations for phases 2 to 4 have been solved numerically (Kamath, 1987), and some simulation results are shown in Fig. 4. From Fig. 4A and 4B, we see that an increase in $k$ leads to a significant increase in the radial growth rate $K_{\mathrm{r}}$ but has essentially no effect either on the rate of height increase $K_{\mathrm{h}}$ or on the final height attained. In this model, the final colony height is a function only of $h_{\mathrm{g}}$ and of $d$. Fig. 4B does not show the plateau for maximum colony height at extended times. From Fig. 4C and 4D, we observe that an increase in $\mu$ results in an increase in both $K_{\mathrm{r}}$ and $K_{\mathrm{h}}$, which is to be expected. A surprising prediction of the model is that there is no effect of $h_{\mathrm{g}}$ and consequently $s_{0}$ on either $K_{\mathrm{r}}$ or $K_{\mathrm{h}}$, although the final colony height increases with increase in $h_{9}$ (Fig. 4E). This is partly corroborated by the experimental finding of this study that $K_{\mathrm{r}}$ is independent of $s_{0}$. Fig. 4F shows the obvious effect of height reached by glucose on the final height of the colony. Also of interest, we found that increasing $h_{0}$ has no effect on either $K_{\mathrm{r}}$ or $K_{\mathrm{h}}$. In summary, this model predicts that the radial growth rate $K_{\mathrm{r}}$ is a function (approximately linear) of the parameter $k$, the depth of oxygen penetration $d$ and the specific growth rate $\mu$, and is insensitive to changes in the initial substrate concentration $s_{0}$ and the initial colony height $h_{0}$. The rate of height increase $K_{\mathrm{h}}$ is a function (approximately linear) of only $d$ and $\mu$. We expected $K_{\mathrm{h}}$ to be independent of $h_{0}$ but did not anticipate that $k$ and $s_{0}$ would have such small effects. 

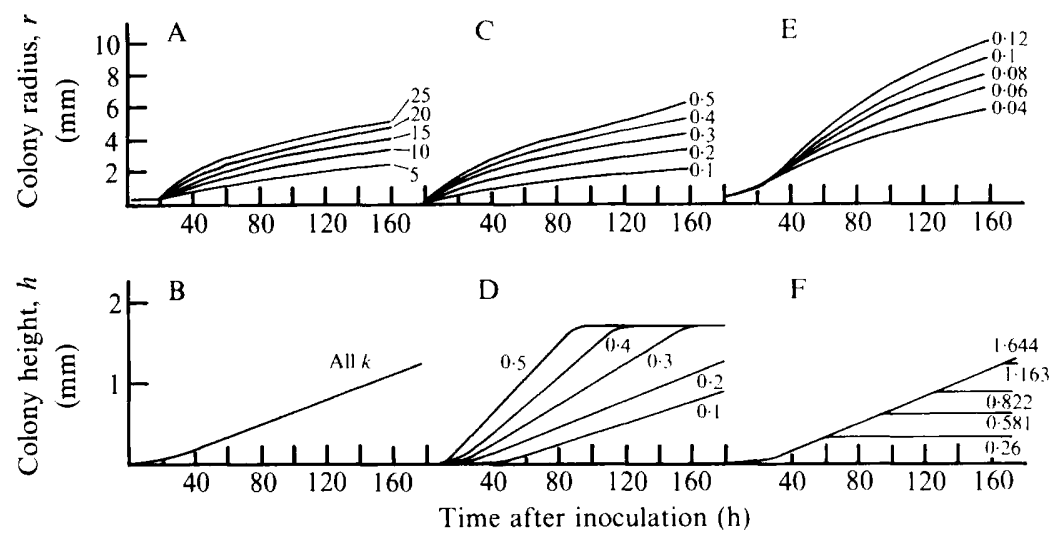

Fig. 4. Simulation results for growth verses time. A, Effect of parameter $k$ on radial growth; numbers refer to $k$ in mm. B, Effect of parameter $k$ on colony height; same $k$ values as for (A). The plateau at 1.78 $\mathrm{mm}$ at extended time is not shown. C, Effect of specific growth rate coefficient $\mu$ on radial growth; numbers refer to $\mu$ in reciprocal $h$. D, Effect of specific growth rate coefficient $\mu$ on colony height; same $\mu$ as (C). E, Effect of oxygen penetration depth $d$, on radial growth; numbers refer to $d$ in mm. F, Effect of height for glucose diffusion $h_{\mathrm{g}}$ on colony height; numbers refer to $h_{\mathrm{g}}$ in $\mathrm{mm}$. The plateau for $1.644 \mathrm{~mm}$ is not shown. Values when held constant: $k=20 ; \mu=0.5 ; d=0.04 ; h_{9}=1.644$; initial colony height assumed $=4 \mu \mathrm{m}$.

Case of varying glucose concentration at agar surface. Here again, it is assumed that the thickness to which a diffusing nutrient can penetrate into the colony is given by equation 17 and that the depth of oxygen penetration $d$ is constant, since for oxygen $s_{0}$ is constant. But the height $h_{\mathrm{g}}$ to which glucose can diffuse varies with time since the glucose concentration at the agar surface directly in contact with the base of the colony $s_{\mathrm{b}}$ is always less than the initial concentration $s_{0}$. This variation in $h_{\mathrm{g}}$ with time makes the problem much more complex than the earlier one, necessitating the quantification of the change in $s_{\mathrm{b}}$ with colony growth. It is assumed that $s_{\mathrm{b}}$ is a function only of the radial distance along the agar surface since Robson et al. (1987) reported that there is no significant variation in the glucose concentration in the agar with depth for fungal colonies. Assuming an expression for the uptake of glucose by the biomass and substituting into the equation for simultaneous diffusion and reaction (equation 6), the change in $s_{\mathrm{b}}$ with changing colony radius can be obtained. In the simplest case of a zero-order uptake, the variation in $s_{\mathrm{b}}$ with radius $r$ is found to be parabolic. A further assumption is that the glucose in the agar that is directly under the colony alone is depleted. This is a reasonable assumption although at very low glucose concentrations Rieck et al. (1973) found it invalid. In this case also, growth can be divided into three or four phases, depending on the conditions of growth.

Cell physiology and metabolism may shift because of ageing and because of inhibition by cell products. The exact effects on supply and demand for glucose and oxygen are beyond the scope of this simple model. Since $h_{\mathrm{g}}$ decreases with time, it is not necessary that $d$ is less than $h_{\mathrm{g}}$ at all times. At this point, it is convenient to define the critical height at which the hypothetical glucose line (actually a curve in this case) touches the tip of the colony as $h_{\mathrm{c}}$. We now have subcase 1 , where $h=d<h_{\mathrm{c}}$ at some region, and subcase 2 , with $h=h_{\mathrm{c}}<d$ at some region, which is likely to occur only at very low glucose concentration. In subcase 1 , exponential growth occurs throughout the colony until the colony height $h$ becomes equal to $d$. This is phase 1 , the exponential phase (Fig. 3A). When $h$ is between $d$ and $h_{\mathrm{c}}$, in phase 2, the growth zone is an annular segment of constant thickness $d$ (Fig. 3B). When $h$ increases beyond $h_{\mathrm{c}}$ and until it reaches the final value of $\left\{h_{g}(t)+d\right\}$, the growth zone is as shown in Fig. 3E. This is phase 3. In the final phase (phase 4), the growth zone is as shown in Fig. 3F. Depending on the uptake rate of the substrate, the tip of the colony may either be flattish or the colony may assume a bimodal shape with two peaks and a valley at the central point of the colony. Thus, this model can accommodate the experimentally observed bimodal nature of some type of colonies. 
In subcase 2, the exponential phase (phase 1) will occur until $h=h_{\mathrm{c}}$. Beyond $h_{\mathrm{c}}$ and until $h=h_{\mathrm{g}}(t)+d$, the growth zone will be as shown in Fig. 3E. In this case, there is a possibility that the $s_{\mathrm{b}}$ may go to zero before the height $h_{\mathrm{g}}(t)+d$ is attained. This is phase 2. Finally, in phase 3, the growth zone will be similar to that in Fig. 3F. Thus, there are only three phases of growth in subcase 2. An appropriate expression for the substrate uptake is needed for solving the above equations using numerical techniques.

Our model for colony growth used the spherical segment approximation for colony shape. Both the simple case of constant glucose concentration at the bottom surface of the colony and the case of nutrient gradients were considered. These models support the experimental findings and help explain the shapes commonly observed with microbial colonies.

\section{REFERENCES}

Allan, E. J. \& Prosser, J. I. (1985). A kinetic study of the colony growth of Streptomyces coelicolor A3(2) and J802 on solid medium. Journal of General Microbiology 131, 2521-2532.

Bull, A. T. \& TrinCI, A. P. J. (1977). The physiology and metabolic control of fungal growth. Advances in Microbial Physiology, 15, 1-84.

Bungay, H. R., Pettit, P. M. \& Drislane, A. M. (1983). Dissolved oxygen contours in Pseudomonas ovalis colonies. American Chemical Society Symposium Series 207, 395-401.

BURCHARD, R. P. (1974). Growth of surface colonies of the gliding bacterium Myxococcus xanthus. Archives of Microbiology, 96, 247-254.

Cooper, A. L., Dean, A. C. R. \& Hinshelwood, C. (1968). Factors affecting the growth of bacterial colonies on agar plates. Proceedings of the Royal Society B171, 175-199.

FralEIGH, S. P. (1985). Strategies for the simulation of nutrient gradients in a microbial colony. MS thesis, Rensselaer Polytechnic Institute, Troy, NY.

Fraleigh, S. P. \& BungaY, H. R. (1986). Modelling of nutrient gradients in a bacterial colony. Journal of General Microbiology 132, 2057-2060.

GraY, B. F. \& KIRWAN, N. A. (1974). Growth rates of yeast colonies on solid media. Biophysical Chemistry 1, 204-213.

Johnson, M. G., Palumbo, S. A., Rieck, V. T. \& WITTER, L. D. (1970). Influence of temperature on steady state growth of colonies of Pseudomonas fiuorescens. Journal of Bacteriology 103, 268-269.

Jones, J. C. \& GraY, B. F. (1978a). Surface colony growth in a controlled nutrient environment 1 . The exponential law. Microbios 22, 185-194.

JoNES, J. C. \& GRaY, B. F. (1978b). Surface colony growth in a controlled nutrient environment 2 . The effect of ethanol. Microbios 22, 195-210.

JoNES, J. C. \& GRAY, B. F. (1979). Surface colony growth in a controlled nutrient environment 3 . Dependence of growth constant on nutrient concentration. Microbios 23, 45-51.

KAMATH, R. S. (1987). Growth of yeast colonies on solid media. MS thesis, Rensselaer Polytechnic Institute, Troy, NY.

LeWIS, M. W. A. \& WimpenNy, J. W. T. (1981). The influence of nutrition and temperatue on the growth of colonies of Escherichia coli K12. Canadian Journal of Microbiology 27, 679-684.
Palumbo, S. A., Johnson, M. G., Rieck, V. T. \& WitTER, L. D. (1971). Growth measurements on surface colonies of bacteria. Journal of General Microbiology 66, 137-143.

Peters, A. C., Wimpenny, J. W. T. \& CoOmbs, J. P. (1987). Oxygen profiles in, and in the agar beneath, colonies of Bacillus cereus, Staphylococcus albus and Escherichia coli. Journal of General Microbiology 133, 1257-1263.

PIRT, S. J. (1967). A kinetic study of the mode of growth of surface colonies of bacteria and fungi. Journal of General Microbiology 47, 181-197.

PIRT, S. J. (1975). Growth of microbiological colonies on the surface of solid medium. In Principles of Microbe and Cell Cultivation. Oxford \& London: Blackwell Scientific Publications.

Prosser, J. I. (1982). Growth of fungi. In Microbial Population Dynamics, pp. 125-166. Edited by M. J. Bazin. Boca Raton: CRC Press.

Rieck, V. T., Palumbo, S. A. \& Witter, L. D. (1973). Glucose availability and the growth rate of colonies of Pseudomonas fluorescens. Journal of General Microbiology 74, 1-8.

Reyrolle, J. \& Letellier, F. (1979). Autoradiographic study of the localization and evolution of growth zones in bacterial colonies. Journal of General Microbiology 111, 399-406.

Robson, G. D., Bell, S. D., KuhN, P. J. \& TRINCI, A. P. J. (1987). Glucose and penicillin concentrations in agar medium below fungal colonies. Journal of General Microbiology 133, 361-367.

WIMPENNY, J. W. T. (1979). The growth and form of bacterial colonies. Journal of General Microbiology 114, 483-486.

WIMPENNY, J. W. T. \& CoOMBS, J. P. (1983). Penetration of oxygen into bacterial colonies. Journal of General Microbiology 129, 1239-1242.

WimpenNY, J. W. T. \& LeWIS, M. W. A. (1977). The growth and respiration of bacterial colonies. Journal of General Microbiology 103, 9-18.

WIMPENNY, J. W. T. \& PARR, J. A. (1979). Biochemical differentiation in large colonies of Enterobacter cloacae. Journal of General Microbiology 114, 487489. 